\title{
Non-invasive markers of airway inflammation and remodeling in childhood asthma
}

\author{
Gagliardo R, La Grutta S, Chanez P, Profita M, Paternò A, Cibella F, \\ Bousquet J, Viegi G, Gjomarkaj M. Non-invasive markers of airway \\ inflammation and remodeling in childhood asthma. \\ Pediatr Allergy Immunol 2009: 20: 780-790. \\ (C) 2009 John Wiley \& Sons A/S
}

To evaluate the relationship between pro-inflammatory and proremodeling mediators and severity and control of asthma in children, the levels of IL-8, MMP-9, TIMP-1 in induced sputum supernatants, the number of sputum eosinophils, as well as FeNO, were investigated in 35 asthmatic children, 12 with intermittent (IA) and 23 with moderate asthma (MA), and 9 controls (C). The patients with asthma were followed for $1 \mathrm{yr}$ and sputum was obtained twice during the follow-up. Biomarker levels were correlated with the number of exacerbations. We found that IL-8, MMP-9, TIMP-1 and the numbers of eosinophils in induced sputum, as well as FeNO, were increased in children with IA and MA in comparison to $\mathrm{C}$. The ongoing inflammation was confirmed by increased nuclear p $65 \mathrm{NF}-\kappa \mathrm{B}$ subunit localization in sputum cells. In MA, FeNO measurements, sputum eosinophils and IL-8 levels, positively correlated with the occurrence of disease exacerbations during a 1-yr follow-up. According to FeNO, sputum eosinophils and IL-8 sputum concentrations, and the number of exacerbations, two distinct phenotypes of MA were identified. This study shows that the presence of bronchial inflammation is detectable in the airways of some IA, as well as in the airways of MA, despite the regular ICS treatment. This study also proposes the need to perform large prospective studies to confirm the importance of measuring specific biomarkers in induced sputum, concomitantly to FeNO analyses, to assess sub-clinical airway inflammation and disease control in children with asthma.

\section{Rosalia Gagliardo ${ }^{1}$, Stefania La Grutta ${ }^{1,2}$, Pascal Chanez ${ }^{3,4}$, Mirella Profita ${ }^{1}$, Alessandra Paternò ${ }^{5}$, Fabio Cibella ${ }^{1}$, Jean Bousquet ${ }^{4}$, Giovanni Viegi ${ }^{1}$ and Mark Gjomarkaj ${ }^{1}$}

${ }^{1}$ Institute of Biomedicine and Molecular Immunology, Unit of Immunopathology and Pharmacology of the Respiratory System, Italian National Research Council, Palermo, Italy, ${ }^{2}$ Allergy Unit of ARNAS and Environmental Health Unit, ARPA, Palermo, Italy, ${ }^{3}$ Département des Maladies Respiratoires, AP-HM, Université de la Méditerranée, 270 boulevard de Sainte-Marguerite, F-13009 Marseille, France, ${ }^{4}$ Institut National de la Santé et de la Recherche Médicale U-454, Clinique des Maladies Respiratoires CHU, Montpellier, France, ${ }^{5}$ DIMPEFINU, Universita' degli Studi di Palermo, Italy

Key words: childhood asthma; inflammation; sputum; exacerbations

Rosalia Gagliardo, PhD, Istituto di Biomedicina e Immunologia Molecolare, Sezione di

Immunopatologia e Farmacologia Clinica e Sperimentale dell'Apparato Respiratorio, Consiglio Nazionale delle Ricerche, Via Ugo La Malfa, 153 - 90146 Palermo, Italy

Tel.: +390916809150

Fax: +39 0916809122

E-mail: gagliardo@ibim.cnr.it

Accepted 12 January 2009

\section{Introduction}

Bronchial asthma is defined as a chronic inflammatory disease of the airways. The presence of chronic inflammation is associated with bronchial obstruction and with structural changes of the airways, i.e. bronchial remodeling (1). The assessment of 'asthma control' as well as the definition of the severity of the disease by the current guidelines are based only on symptoms, short-acting $\beta_{2}$-agonists use, and on

Abbreviations: FeNO, exhaled nitric oxide; IL-8, interleukin-8; MMP-9, metalloproteinase-9; TIMP-1, tissue inhibitor metalloproteinase-1; NF-кB, nuclear factor kappa B. measures of lung function (2). No inflammatory parameters are presently taken into account although often symptoms are not specific and not closely related to the presence and severity of airway inflammation (3).

Some interesting studies have been aimed to define the inflammatory and immunologic components of asthma in children $(4,5)$, however relatively few data are available on the definition of the biologic mechanisms underlying inflammation and remodeling in these asthmatic patients using non-invasive methods currently available, such as exhaled nitric oxide (FeNO) and induced sputum $(6,7)$. Some studies have addressed the usefulness of FeNO and induced 
sputum in detecting airway inflammation in asthmatic patients (7-10). FeNO is associated to respiratory symptoms, lung function, bronchial responsiveness, blood eosinophilia and it has been already demonstrated, both in adults and children, that a strategy based on the measurement of FeNO or sputum eosinophilia allows to avoid asthma exacerbations, to reduce inhaled corticosteroids requirement and to improve airway hyperresponsiveness $(11,12)$.

Most of the mediators related to asthma inflammation and remodeling, including Interleukin-8 (IL-8), Metalloproteinases (MMPs), and inhibitors of metalloproteinase (TIMPs), can be measured in the supernatant of induced sputum $(13,14)$. IL-8, has been shown to play an important role in adult and childhood asthma, and despite continuous long-term treatment with systemic and inhaled glucocorticoids (GC), high levels of IL-8 are synthesized by airway cells and peripheral blood mononuclear cells (PBMC) of severe uncontrolled asthmatics $(15,16)$, and moderate persistent asthmatic children where IL-8 is correlated with the occurrence of exacerbations (6). MMPs and TIMPs, are important mediators of inflammation, cytokine activation, and tissue remodeling. The balance between MMPs and the TIMPs plays an important role in the breakdown and deposition of extra-cellular matrix (ECM) in the airways wall representing an important cause of airflow limitation in asthma (17). Although the major source of MMP-9 in the lung is represented by macrophages, followed by neutrophils and epithelial cells, high levels of active MMP-9 have been also demonstrated to be released by eosinophils in asthmatic airway inflammation (18) and in eosinophilic asthma (19). IL-8 has been shown to induce the release of MMP-9 from neutrophils, and in turn MMP-9 can increase IL-8 potency (20). In this context, it was observed that the transcriptional factor $\mathrm{NF}-\kappa \mathrm{B}$, which plays a role in the persistence of inflammation (21), regulates IL-8 (22) and MMP-9 (23) gene expression and is in part responsible (via IKK/NF- $\mathrm{KB}$ pathway) of the TIMP-1 gene regulation (24).

We hypothesized that markers of inflammation or remodeling in induced sputum may mirror asthma severity and control in asthmatic children. For this reason, we undertook a prospective follow-up study in children with intermittent asthma (IA), and moderate asthma (MA), who were clinically and functionally well controlled and were receiving medium doses of inhaled corticosteroids and long-acting $\beta_{2}$ agonists. We aimed to investigate the inflammatory status' of these patients using non-invasive methods including induced sputum biomarkers measurements (number of eosinophils, IL-8, MMP-9, TIMP-1 and p65 NF- $\mathrm{B}$ subunit), concomitantly with FeNO. Finally, we evaluated whether these biomarkers were correlated with the development of disease exacerbations throughout an 1-yr follow-up period of time.

\section{Materials and methods}

Subjects

Forty-three asthmatics (6-14 yr, mean s.d.: $9.2 \pm 2.5$ ) were recruited from the outpatient clinic of the children hospital ARNAS in Palermo, Italy. The diagnosis of asthma and the assessment of its severity and control were done at study entry according to Global Initiative for Asthma (25). Only 35 of 43 asthmatic children were able to obtain suitable sputum samples at visit 1 and to be included in the study. All these included subjects were able to perform induced sputum at visit 3 , either on the scheduled day of visit 3 or within $1 \mathrm{wk}$ from it.

Twelve children had intermittent asthma, treated with short-acting $\beta_{2}$ agonists on demand during the last 3 months; 23 children had moderate asthma treated with fluticasone propionate $250 \mu \mathrm{g}$ [inhaler device Diskus (GlaxoSmithKline, Brentford, UK)] plus Salmeterol $50 \mu \mathrm{g}$ bid, for at least 3 months. This high dose of Fluticasone was used to stabilize these patients and to let them have a 'controlled' disease, at least considering the clinical and functional parameters. The control group consisted of nine healthy children. Throughout a longitudinal 1-yr follow-up study, the visits $1,2,3$ and 4 were scheduled every 4 months. The study was approved by the Institutional Ethical Committee. All parents of the children signed a written informed consent.

Clinical assessment and compliance of patients

Pulmonary function tests were performed as recommended by the American Thoracic Society (26). Forced expiratory volume in $1 \mathrm{~s}\left(\mathrm{FEV}_{1}\right)$, forced vital capacity (FVC) were measured according to ATS guidelines and the best of three technically acceptable and reproducible maneuvers was retained (26). Atopic status was assessed by skin-prick tests (27) to aeroallergens commonly present in the Mediterranean area, and total serum $\mathrm{IgE}$ measurements. The compliance to treatment was assessed by checking the inhalation technique at each visit. We also measured basal plasma cortisol concentrations at 8:00 AM by electrochemiluminescence twice (visits 1 and 4). Results were 
expressed in nanomolar concentrations, and adherence to inhaled corticosteroids was considered satisfactory if cortisol was less than $100 \mathrm{~nm}$ $(28,29)$. To accurately monitor the patients' symptoms, at the beginning of the study parents were provided with cell phone numbers of phisicians who were on call always reachable $24 \mathrm{~h}$ a day for the entire follow-up period. Parents were accurately instructed to refer to physicians about any symptoms occurred. When symptoms possibly related to putative exacerbations occurred, on the basis of physician's evaluation, patients underwent unscheduled visits on the same or on the following day, in order to verify the effective presence and to classify and treat the exacerbations. Asthma exacerbations were defined as increasing symptoms and signs of asthma (coughing, wheezing, shortness of breath) unresponsive to the patient's routine asthma medication and requiring an unscheduled visit. Mild and severe exacerbations were differentiated according to the Formoterol and Corticosteroids Establishing Therapy study (30). Severe exacerbations were treated with a short course of oral steroids $(1 \mathrm{mg} /$ $\mathrm{kg}$ of prednisolone for 2-5 days). The number of mild and severe exacerbations occurred during the follow-up period was recorded. Moderate asthmatic children were divided into two subgroups on the basis of the frequency of severe exacerbations (31).

\section{Nitric oxide measurement}

FeNO was determined by a chemiluminescence analyzer connected to a computerized system (NIOX; Aerocrine AB, Solna, Sweden), and measured in triplicate after fast inhalation maneuvers to total lung capacity, at a constant flow rate of $50 \mathrm{ml} / \mathrm{s} \mathrm{(32).} \mathrm{The} \mathrm{mean} \mathrm{of} \mathrm{three}$ measures was retained for the analysis.

\section{Sputum induction}

Each subject underwent spirometry before the beginning of the procedure. If $\mathrm{FEV}_{1}$ was over $75 \%$ of predicted at baseline and the child had not used a short-acting $\beta 2$-agonist within the last $6 \mathrm{~h}$, we performed the procedure without premedication. Patients were exposed to an aerosol of $4 \%$ hypertonic saline solution, monitoring the assessment of bronchial reactivity, as described elsewhere (33).

\section{Sputum processing}

Sputum processing was performed according to the methods of Hargreave et al. with minor modifications (34). The cells obtained from induced sputum were then cytocentrifuged (Cytospin 2; Shandon Instruments, Runcorn, UK) and stained with Diff-Quick (Merz-Dade, Dudingen, Switzerland $(\mathrm{CH})$ ). Slides were read blindly by two independent investigators and differential cell counts were expressed as a percentage of 400 cells.

Measurement of IL-8, MMP-9, TIMP-1

Evaluation of IL-8, MMP-9, and TIMP-1 concentrations in induced sputum supernatants was assessed using commercially available specific enzyme-linked immunoadsorbent assay kits (R\&D Systems, UK; Biotrack, Amersham International plc, UK) according to the manufacturer's instructions.

\section{Immunocytochemistry analysis}

To further characterize the ongoing bronchial inflammation in both groups of asthmatics, we performed immunocytochemical analyses in sputum cells to determine the subcellular distribution of p65 NF- $\mathrm{B}$ subunit. After thawing, immunoreactivity for $\mathrm{p} 65 \mathrm{NF}-\kappa \mathrm{B}$ subunit, was evaluated using the labeled streptavidin-biotin method (Alkaline phosphatase Rabbit/Mouse/ Goat; Universal LSAB + kit; Dako, Glostrup, Denmark), as previously described (21). The cell identification was based on cell morphology under light microscopy $(\times 400$ final magnification), carefully referring to the cell type distribution in corresponding Diff-Quick-stained slides. p65 localization was expressed as percentage of sputum cells showing a p65 nuclear staining. Goat polyclonal anti-p65 NF-kB subunit (Santa Cruz Biotechnology, Santa Cruz, California) was diluted 1:20.

\section{Study design}

After 1 month run-in period, during which $\mathrm{FEV}_{1}$ before and after broncho-dilation $(400 \mu \mathrm{g}$ of salbutamol) was assessed, patients attended the outpatient clinic (visit 1) and underwent clinical assessment (pre- and postbronchodilator FEV $\mathrm{F}_{1}$ ) after 4 (visit 2), 8 (visit 3) and 12 (visit 4) months by the same physician (S.L.G) (Fig. 1). In patients with an upper respiratory tract infection, follow-up visits were postponed for $2 \mathrm{wk}$. Biomarkers and FeNO levels were measured at visit 1 and at visit 3. Sputum eosinophilia, IL-8, MMP-9, and TIMP-1 sputum concentrations were measured in nine controls (C), 12 IA and 23 MA children. p65 nuclear localization was 


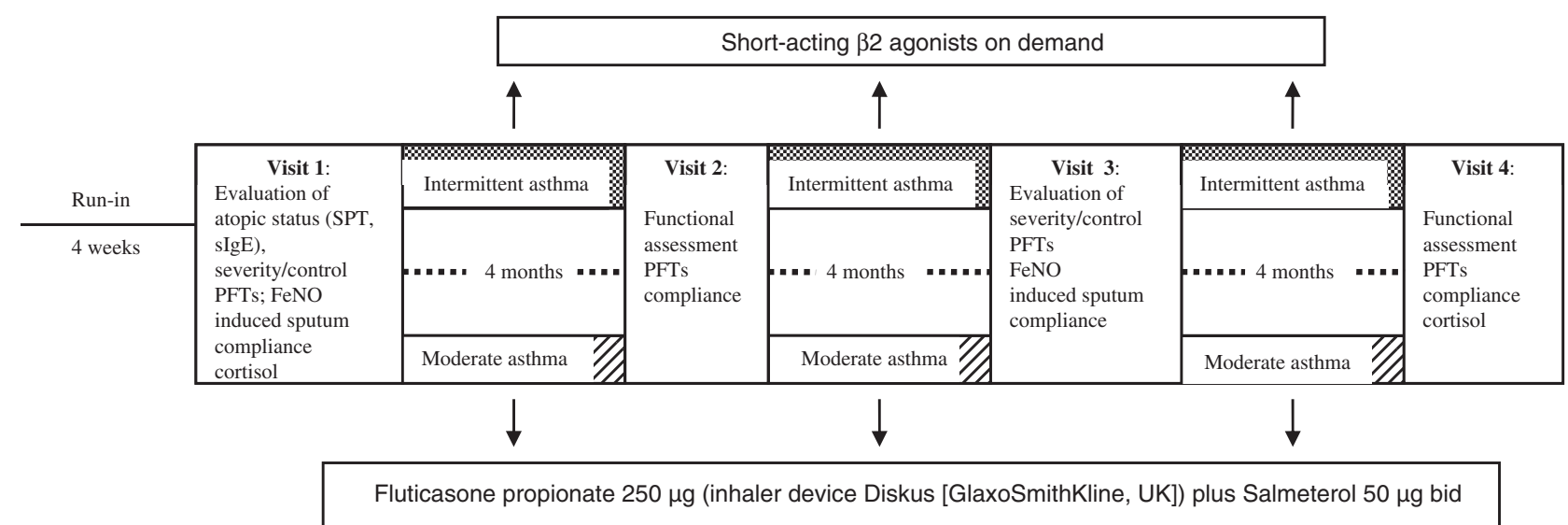

Fig. 1. Flow chart of the study.

evaluated in group subsets, owing to limitation in the amount of sputum cells recovered, in 6 out of 9 C, 8 out of 12 IA and 12 out of 23 MA children.

\section{Statistical analysis}

As variables were normally distributed, analysis of variance (ANOVA) was used for between-group comparisons with Bonferroni's correction for multiple comparisons. Correlations were analyzed by linear regression analysis and multiple linear regression analysis model.

\section{Results}

Demographic and functional characteristics of patients

The demographic and functional characteristics of patients are reported in Table 1. No statistically significant differences were found among the three study groups in terms of $\mathrm{FEV}_{1}$ values at all visits indicating that, on the basis of this parameter, the asthmatic patients were functionally under control. We observed a significant $\mathrm{FEV}_{1} / \mathrm{FVC}$ decline in MA in comparison with $\mathrm{C}$, and in MA in comparison with IA at all visits. In

Table 1. Demographic, functional and cellular characteristics of subjects

\begin{tabular}{|c|c|c|c|c|c|c|}
\hline & \multirow[b]{2}{*}{ Controls } & \multirow{2}{*}{$\frac{\text { Intermittent }}{\text { Asthma }}$} & \multirow{2}{*}{$\begin{array}{c}\text { Moderate } \\
\text { Asthma }\end{array}$} & \multicolumn{3}{|c|}{$\mathrm{p}$-value } \\
\hline & & & & $\mathrm{C} / \mathrm{IA}$ & $\mathrm{C} / \mathrm{MA}$ & $\mathrm{IA} / \mathrm{MA}$ \\
\hline Number & 9 & 12 & 23 & & & \\
\hline Sex, M/F & $6 / 3$ & $8 / 4$ & $15 / 8$ & & & \\
\hline Age & $10(9-10)$ & $9(8-10)$ & $11.5(10-12)$ & NS & NS & NS \\
\hline Total lgE (KU) & 20 (15-20) & 522 (308-857) & $536(387-770)$ & $p<0.002$ & $p<0.001$ & NS \\
\hline $\mathrm{FEV}_{1}(\%)$ visit1 & $101(100-103)$ & 98 (94.5-104) & 92 (89-96) & NS & NS & NS \\
\hline $\mathrm{FEV}_{1}(\%)$ visit2 & $105(100-110)$ & 97 (92.5-102) & 92 (87-98) & NS & NS & NS \\
\hline $\mathrm{FEV}_{1}(\%)$ visit3 & $100(100-102)$ & $97(92-100)$ & 87 (78-94) & NS & NS & NS \\
\hline $\mathrm{FEV}_{1}(\%)$ visit4 & $102(100-106)$ & 100 (89-104) & 88 (84-94) & NS & NS & NS \\
\hline $\mathrm{FEV}_{1} / \mathrm{FVC}(\%)$ vis1 & 100 (98.5-105) & 94 (86-109) & 83 (75-86) & NS & $p<0.0001$ & $p=0.0005$ \\
\hline $\mathrm{FEV}_{1} / \mathrm{FVC}(\%)$ vis2 & 103 (102-107) & 92 (89-99) & $87.5(84-91)$ & $p=0.01$ & $p<0.0001$ & $p=0.01$ \\
\hline $\mathrm{FEV}_{1} / \mathrm{FVC}(\%)$ vis3 & $100(100-106)$ & 93 (90-98) & 85 (81-88) & NS & $p<0.0001$ & $p=0.002$ \\
\hline $\mathrm{FEV}_{1} / \mathrm{FVC}(\%)$ vis4 & 105 (100-106.5) & 92 (89-99) & $85(80-89)$ & $p=0.01$ & $p<0.0001$ & $p=0.002$ \\
\hline FeNO visit1 & $8(6-10)$ & $18(13-36)$ & 22 (16-30) & $p=0.0085$ & $p=0.013$ & NS \\
\hline FeNO visit3 & $9(7-11)$ & 19.5 (13.5-26) & $22.5(14-30)$ & $p=0.0066$ & $p=0.0023$ & NS \\
\hline Sputum eosinophils visit1 & 0 & $5.75(3-8)$ & $4(3-9)$ & $p=0.0015$ & $p=0.0026$ & NS \\
\hline Sputum eosinophils visit3 & $0.1(0-0.5)$ & $4.9(3-7)$ & $4.5(3.5-7)$ & $p=0.0033$ & $p=0.0036$ & NS \\
\hline Asthma duration, yr & NA & $4(3.25-6)$ & $6(4.25-6.75)$ & & & NS \\
\hline Total exacerbations & 0 & $2(2-3)$ & $3(2-4)$ & & & $p=0.01$ \\
\hline Severe exacerbations & 0 & $1(0-0)$ & $2(1-2.5)$ & & & $p<0.0001$ \\
\hline Cortisol $(\mathrm{nm}) \dagger$ & $346(215-458)$ & $194(170-225)$ & $66(58-95)$ & $p<0.001$ & $p<0.0001$ & $p<0.004$ \\
\hline Cortisol $(\mathrm{nm}) \ddagger$ & $342(215-447)$ & $195(170-225)$ & 69 (56-95) & $p<0.002$ & $p<0.0001$ & $p<0.004$ \\
\hline
\end{tabular}

Results are expressed as median and (25-75 percentiles).

$\uparrow$ Cortisol levels at study entry.

$\$$ Cortisol levels at the end of the follow-up period (12 months). 
IA a significant $\mathrm{FEV}_{1} / \mathrm{FVC}$ decline in comparison to $\mathrm{C}$ was observed only at visits 2 and 4 (Table 1). In IA 25 exacerbations occurred (mean 2.1 exacerbations per patient), 24 of which mild and 1 severe. In MA 73 exacerbations occurred (mean 3 exacerbations per patient), 43 of which mild and 30 severe.

\section{FeNO and eosinophil number measurements}

The levels of FeNO and the number of sputum eosinophils were significantly increased in IA and MA in comparison with $\mathrm{C}$ at both visits 1 and 3, whilst no significant differences were detected between the two groups of asthmatics (Table 1).

\section{Detection of IL-8, MMP-9, TIMP-1}

We measured the concentrations of IL-8, MMP-9, and TIMP-1 in the induced sputum supernatants recovered from the three groups of subjects at visits 1 and 3. The concentrations of IL-8 (Fig. 2), MMP-9 and TIMP-1 (Fig. 3, a-b) were significantly increased in the two groups of asthmatics when compared with controls at both visits 1 and 3. No significant differences were detected in terms of MMP-9/TIMP-1 molar ratio at both visits 1 and 3 among the three study groups (Fig. 3c). No significant differences were present comparing the levels of each specific mediator at visit 1 and 3 (Figs 2 and 3).

Total and differential cell counts in induced sputum samples

The percentage of eosinophils was significantly higher in IA and MA than in $\mathrm{C}(\mathrm{p}<0.0005)$ (Table 2). The IA and MA also had a significantly increased percentage of neutrophils and a significantly decreased percentage of macrophages when compared with $\mathrm{C}$ (Table 2). The median viability of the cells obtained from induced sputum samples was $78 \%$ (25 to 75 percentiles: 70 to 85 ) in



Fig. 2. Concentrations of interleukin-8 (IL-8) in sputum supernatants of intermittent and moderate asthmatic children and controls measured at visit 1 and visit 3 . Individual data are shown. Horizontal lines represent the median.
$\mathrm{C}$ and $72 \%$ (25 to 75 percentiles: 65 to 80 ) in asthmatic children.

Subcellular distribution of p65 NF-kB subunit

The percentage of induced sputum cells with a p65 nuclear staining (p65 localization was expressed as percentage of sputum cells showing a p65 nuclear staining) was significantly higher in IA and MA than in C ( $<0.0005)$ (Table 3).

The percentage of macrophages, eosinophils and neutrophils of sputum cells with a positive p65 nuclear staining was significantly higher in IA and MA than in C, at both visits 1 and 3 . No differences were detected between the two groups of asthmatic patients (Table 3 ).

\section{Correlations}

IL-8 sputum concentrations positively correlated with MMP-9 and with TIMP-1 $(\mathrm{p}<0.0001$,
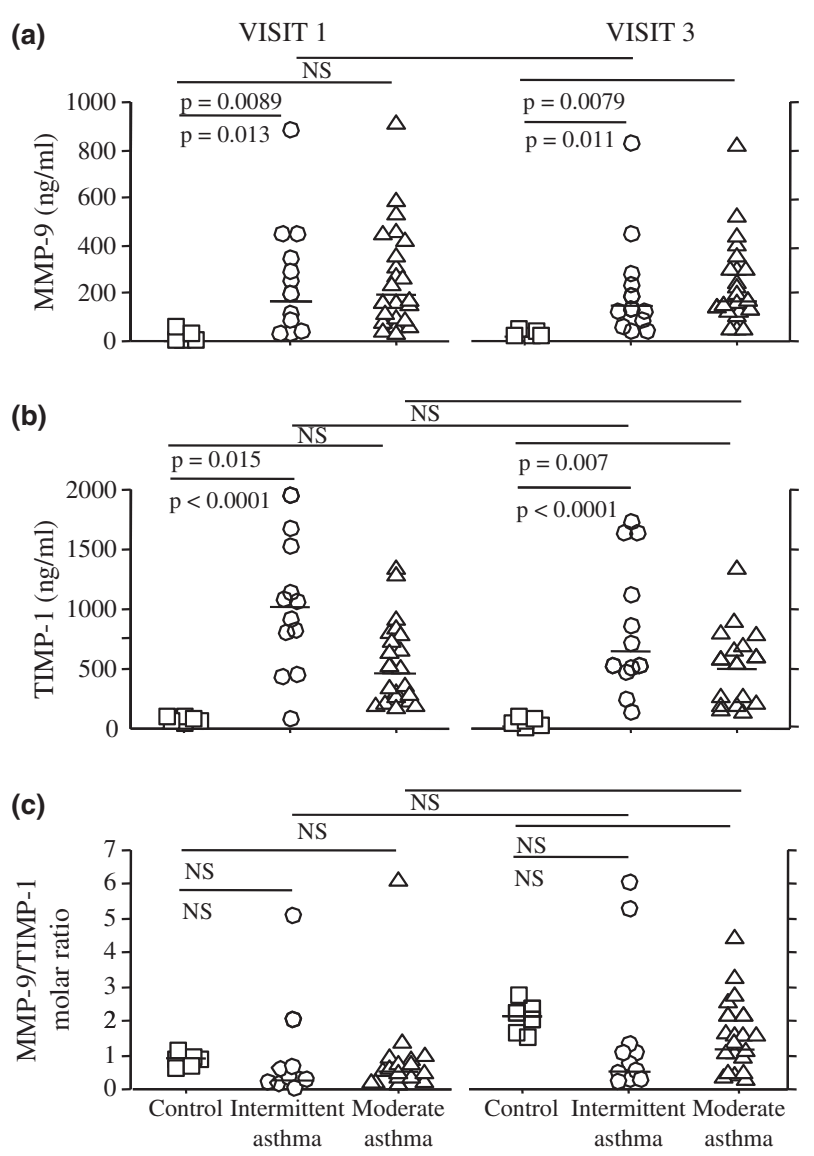

Fig. 3. Concentrations of metalloproteinase-9 (MMP-9) (a), and tissue inhibitor metalloproteinase-1 (TIMP-1) (b) in sputum supernatants of intermittent and moderate asthmatic children and controls, measured at visit 1 and visit 3 . (c) MMP-9/TIMP-1 molar ratio in sputum samples of the three study groups. Individual data are shown. Horizontal lines represent the median. 
Table 2. Total and differential count of cells from induced sputum

\begin{tabular}{|c|c|c|c|c|c|c|c|c|c|c|c|c|}
\hline & \multicolumn{2}{|c|}{ Control subjects } & \multicolumn{2}{|c|}{ Intermittent asthma } & \multicolumn{2}{|c|}{ Moderate asthma } & \multicolumn{6}{|c|}{$p$-value } \\
\hline & \multirow[b]{2}{*}{ vis 1} & \multirow[b]{2}{*}{ vis 3} & \multirow[b]{2}{*}{ vis1 } & \multirow[b]{2}{*}{ vis 3} & \multirow[b]{2}{*}{ vis 1} & \multirow[b]{2}{*}{ vis 3} & \multicolumn{2}{|c|}{$\mathrm{C} / \mathrm{IA}$} & \multicolumn{2}{|c|}{$\mathrm{C} / \mathrm{MA}$} & \multicolumn{2}{|c|}{ IA/MA } \\
\hline & & & & & & & vis 1 & vis 3 & vis 1 & vis 3 & vis 1 & vis 3 \\
\hline Total cell counts $\left(10^{6}\right.$ cells $\left./ \mathrm{ml}\right)$ & $0.8(0.5-1.5)$ & $0.7(0.4-1.2)$ & $1.5(0.8-1.9)$ & $1.1(0.8-1.7)$ & $1.2(0.6-1.8)$ & $1(0.7-2)$ & NS & NS & NS & NS & NS & NS \\
\hline Squamous cells (\%) & $3(2-5)$ & $3.5(1.5-4.5)$ & $2(1-4)$ & $1.5(1-2.5)$ & $2(0-3)$ & $1.5(1-2.75)$ & NS & NS & NS & NS & NS & NS \\
\hline Macrophages (\%) & $91(90-95)$ & $89.5(89-95)$ & $56.5(49.5-70.6)$ & $54(50-71)$ & $52.5(25-63)$ & $49.3(21-60)$ & ** & $* *$ & $* *$ & ** & NS & NS \\
\hline Eosinophils (\%) & 0 & $0.1(0-0.5)$ & $5.75(3-8)$ & $4.9(3-7)$ & $4(3-9)$ & $4.5(3.5-7)$ & ** & $* *$ & ** & ** & NS & NS \\
\hline Neutrophils (\%) & $9(5-11)$ & $10(5-13)$ & $33.5(6-36)$ & $35.5(16-45)$ & $40(12-61)$ & $42(6.75-56.75)$ & $*$ & * & $* *$ & $* *$ & NS & * \\
\hline Lymphocytes (\%) & $0(0-0.5)$ & $0(0-0.8)$ & $3.5(1-3)$ & $4(2.1-6)$ & $2.5(1.5-4.5)$ & $3(2-4.5)$ & NS & * & NS & * & NS & NS \\
\hline Epithelial cells (\%) & $0(0-2.6)$ & $0.5(0-1.8)$ & $0.5(0-2)$ & $1.5(1-2)$ & $1(0-1.8)$ & $1.2(0-2)$ & NS & NS & NS & NS & NS & NS \\
\hline
\end{tabular}

Results are expressed as median (25-75 percentiles) of positive cells with respect to total cell counts.

${ }^{*} p<0.005 ;{ }^{* *} p<0.0005$; NS, not significant.

$\mathrm{R}^{2}=0.66 ; \mathrm{p}=0.004, \mathrm{R}^{2}=0.4$, respectively) in MA. In this group of subjects, a positive correlation was also present between the number of sputum eosinophils and MMP-9 and TIMP-1 $\left(\mathrm{p}=0.04, \quad \mathrm{R}^{2}=0.25 ; \quad \mathrm{p}=0.01, \quad \mathrm{R}^{2}=0.3\right.$, respectively).

The number of severe exacerbations occurred in MA during the follow-up significantly and positively correlated with the levels of FeNO (Fig. 4a), the number of eosinophils (Fig. 4b), and the sputum concentrations of IL-8 at both visits 1 (Fig. 4c) and 3 (data not shown). When a multiple linear regression analysis was performed between severe exacerbations as dependent variable and FeNO, eosinophils and IL-8 as independent variables, the $\mathrm{R}^{2}$ value showed a marked increase (0.79) compared with single linear regressions. On the basis of the number of severe exacerbations occurred during the followup, it was possible to identify two distinct subgroups of patients with MA classified as frequent exacerbators $(\geq 2$ exacerbations, $\mathrm{n}=10)$ and infrequent exacerbators $(0$ or $<2$ exacerbations, $n=13$ ). The FeNO levels (Fig. 4d), the number of sputum eosinophils (Fig. 4e) and the IL-8 sputum concentrations
(Fig. 4f) measured in the frequent exacerbators were significantly higher than in the infrequent exacerbators. No significant correlation was found between MMP-9, TIMP-1, and the MMP-9/TIMP-1 molar ratio and the number of severe exacerbations.

The number of sputum eosinophils (data not shown), and IL-8 concentrations (Fig. 5a) positively correlated with the asthma duration $\left(\mathrm{p}=0.04, \quad \mathrm{R}^{2}=0.25 ; \mathrm{p}=0.01, \quad \mathrm{R}^{2}=0.25\right.$, respectively), while IL-8 concentrations inversely correlated with $\mathrm{FEV}_{1} / \mathrm{FVC} \quad(\mathrm{p}=0.01$, $\mathrm{R}^{2}=0.36$ ) (Fig. 5b) in MA. In addition, when IA and MA were grouped together, the number of total exacerbations, regardless severity, at both visits 1 and 3, significantly correlated with sputum eosinophilia $\left(\mathrm{p}=0.03 ; \quad \mathrm{R}^{2}=0.4\right.$; $\mathrm{p}=0.04 ; \mathrm{R}^{2}=0.36$, respectively), and IL-8 levels $\left(\mathrm{p}=0.01 ; \mathrm{R}^{2}=0.43 ; \mathrm{p}=0.02 ; \mathrm{R}^{2}=\right.$ 0.4 , respectively), but not with FeNO levels (data not shown). No significant correlations were found between total number of exacerbations and MMP-9, TIMP-1, and MMP-9/TIMP-1 molar ratio. MMP-9 positively correlated with IL-8 levels $(\mathrm{p}<0.0001$, $\left.\mathrm{R}^{2}=0.46\right)$.

Table 3. Subcellular distribution of p65 NF-kB subunit by different cell types

\begin{tabular}{|c|c|c|c|c|c|c|c|c|c|c|c|c|}
\hline & \multicolumn{2}{|c|}{ Control subjects } & \multicolumn{2}{|c|}{ Intermittent asthma } & \multicolumn{2}{|c|}{ Moderate asthma } & \multicolumn{6}{|c|}{ p-value } \\
\hline & \multirow[b]{2}{*}{ vis 1} & \multirow[b]{2}{*}{ vis 3} & \multirow[b]{2}{*}{ vis1 } & \multirow[b]{2}{*}{ vis 3} & \multirow[b]{2}{*}{ vis 1} & \multirow[b]{2}{*}{ vis 3} & \multicolumn{2}{|c|}{$C / I A$} & \multicolumn{2}{|c|}{$\mathrm{C} / \mathrm{MA}$} & \multicolumn{2}{|c|}{ IA/MA } \\
\hline & & & & & & & vis 1 & vis 3 & vis 1 & vis 3 & vis 1 & vis 3 \\
\hline Total cells & $2(0-2.5)$ & $2.3(0-4)$ & $54(40-68)$ & $59(39-77)$ & $60(55-67)$ & $58(50-72)$ & $* *$ & $* *$ & $* *$ & ** & NS & NS \\
\hline Macrophages (\%) & $1.5(0-2.5)$ & $1(0-1.75)$ & $42(38-45)$ & $44(40-51)$ & $39(30-50)$ & 40 (33-49) & $* *$ & $* *$ & $* *$ & $* *$ & NS & NS \\
\hline Eosinophils (\%) & $0(0-0)$ & $0(0-0)$ & $5(3-8)$ & $6.5(2-10)$ & $7(5-9)$ & $6.1(4.5-8.5)$ & $* *$ & $* *$ & $* *$ & $* *$ & NS & NS \\
\hline Neutrophils (\%) & $1(0-2)$ & $0.5(0-1.75)$ & $7(6-11)$ & $9(6-12)$ & $13(9-15)$ & $10(7.5-14.5)$ & $* *$ & $* *$ & ** & ** & NS & NS \\
\hline Lymphocytes (\%) & $0(0-0)$ & $0(0-0.5)$ & $1(0-2)$ & $1.2(0.75-1.75)$ & $1(0-1.5)$ & $2(0-2.75)$ & NS & * & NS & * & NS & NS \\
\hline
\end{tabular}

Results are expressed as median (25-75 percentiles) of sputum cells with a p65 nuclear staining with respect to total cell counts.

${ }^{*} p<0.005 ;{ }^{* *} p<0.0005$; NS, not significant. 


\section{Gagliardo et al.}
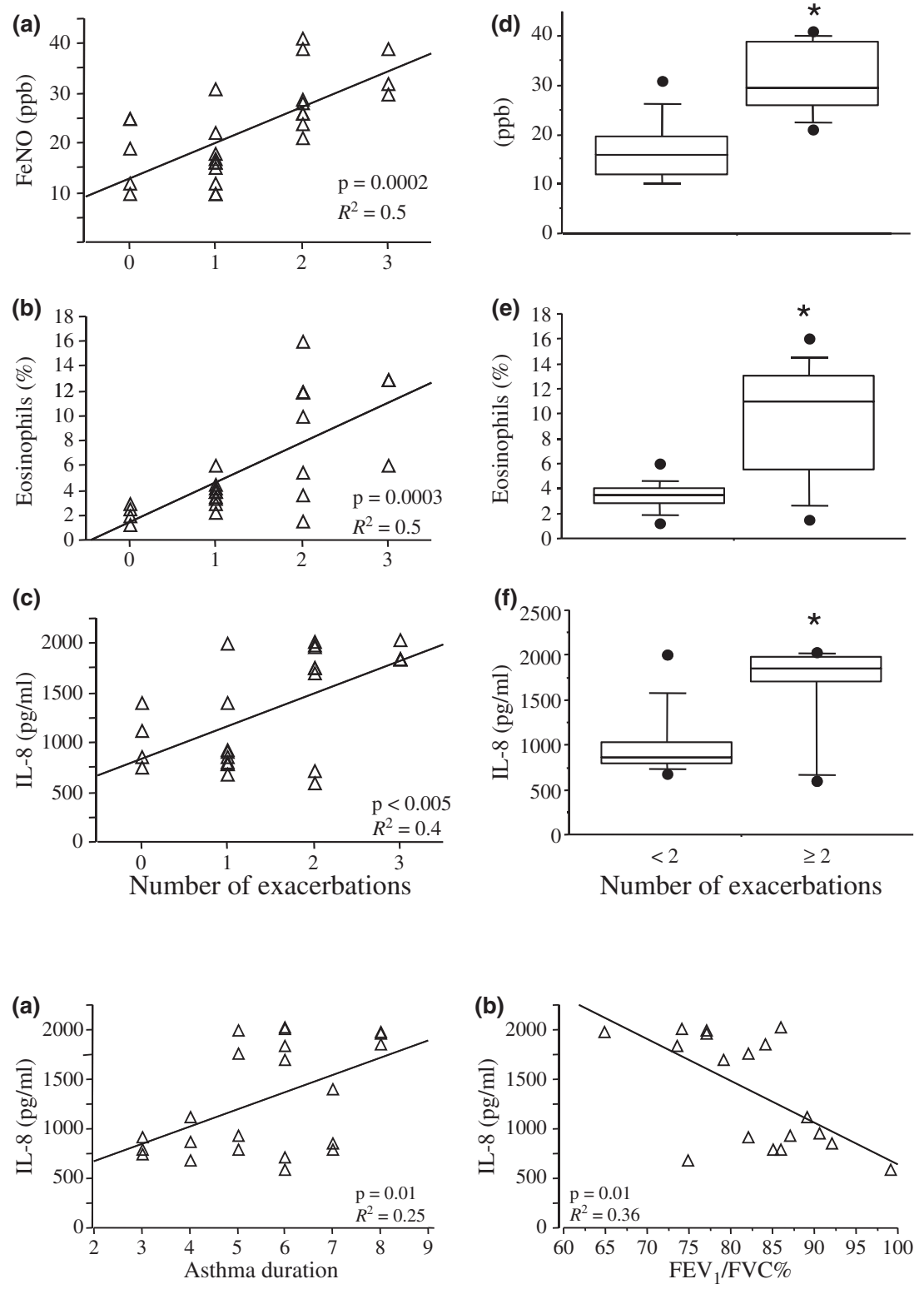

Number of exacerbations
Fig. 4. Left panels: Correlation

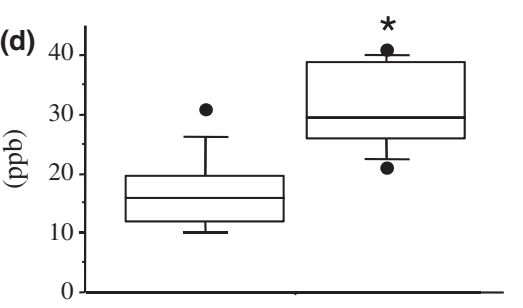

between number of exacerbations and FeNO levels (a), number of sputum eosinophils (b), and sputum IL-8 concentrations (c) in moderate asthmatic children at visit 1 .

Correlations were calculated by linear regression analyses. Right panels: FeNO levels (d), number of sputum eosinophils (e), and sputum IL-8 concentrations (f) in moderate asthmatic children grouped as frequent $(\geq 2$ exacerbations, $\mathrm{n}=10)$ and infrequent exacerbators ( 0 or $<2$ exacerbations, $n=13$ ). Bars indicate (from the bottom to the top) 10th, 25th, 50th (median), 75th, and 90th percentiles. Values below 10th and above 90th percentiles are plotted as circles.

Fig. 5. Correlations between sputum IL-8 concentrations and asthma duration (a), and between sputum IL-8 levels and $\mathrm{FEV}_{1} / \mathrm{FVC} \%$ (b), in moderate asthmatic children. Correlations were calculated by linear regression analyses.

\section{Discussion}

This study shows that the levels of several biomarkers, including IL-8, MMP-9, TIMP-1, number of eosinophils in induced sputum as well as the levels of FeNO, are increased in children with IA and MA as compared to $\mathrm{C}$ and that, according to the levels of FeNO, number of eosinophils, and IL-8 sputum concentrations, two different phenotypes of MA children can be identified. These three latter markers correlate, in MA, with the occurrence of disease exacerbations during a 1-yr follow-up period of time.

Treatment with ICS reduces FeNO by a direct effect on transcription of inducibile NO synthase $(35,36)$ and, accordingly, FeNO has been proposed as a sensitive non-invasive and reproduc- ible marker of asthma activity that well correlates with eosinophilic airway inflammation (37). These findings suggest the possibility to use this methodology in children to monitor the efficacy of the therapy on this specific parameter, although recent studies showed that a therapy regimen aimed to lowering $\mathrm{FeNO}$ in children with asthma improved parameters of small airways function but was not able to improve clinical markers of asthma control (38). In this context, we observed increased levels of FeNO and sputum eosinophils in children with IA and MA when compared with $\mathrm{C}$ while no significant differences were observed between both groups of asthmatics during the 1-yr follow-up. Since it has been demonstrated a heterogeneity of FeNO 
response to ICS (39) and since FeNO indicates the severity and the control of childhood asthma (40) the observed absence of a significant difference in oral FeNO between children with IA and MA demonstrates that, despite the use of ICSs, increased levels of oxidative stress markers, as well as elevated eosinophils levels, persist in some of these patients. Our findings that FeNO is increased in a subgroup of MA patients confirm previous observations and extend them providing new information on the usefulness of the use of this marker in monitoring bronchial inflammation in asthma. Moreover, these findings support the concept of the presence of different phenotypes of asthmatic patients detectable using noninvasive methods including FeNO measurement.

IL-8 has been shown to play an important role in the airways and in the systemic circulation of both adulthood $(16,41)$, and childhood asthma (6). High levels of IL-8 are synthesized from PBMC of adult severe asthmatics (16), and moderate persistent asthmatic children with significant differences when compared with intermittent asthmatic children (6). An increase in the expression of IL-8 was observed in the airway mucosa of adult moderate-to-severe asthmatics (41) as well as in the induced sputum supernatants of adult severe asthmatics showing an elevated grade of neutrophilia (15) despite these patients were receiving ICS therapy (41). In addition, increased levels of IL-8 were observed in induced sputum (42) of children with acute asthma. In this context there were not clear evidences about the effects of ICS on the reduction of the levels of IL-8 in the airways of asthmatic children. The present study extends these findings at bronchial levels of IA and MA children showing increased levels of IL-8 in induced sputum supernatants when compared with C. Although no significant differences were observed between IA and MA children during the follow-up, we observed the presence of two sub-groups of MA children: one showing a reduction of IL-8 levels and another group showing the presence of high levels of IL-8 suggesting the presence of bronchial inflammation despite the regular ICS treatment. These observations support the concept of heterogeneity of bronchial asthma in MA children $(6,39)$ and, once again, suggest the presence of different phenotypes of MA children.

Airway remodeling, traditionally referred to the advanced steps of the disease (14), has been shown to be present very early in the course of childhood asthma (43). For this reason, it is important to assess the relationships between airway inflammation and remodeling and the clinical spectrum of asthma severity in children. We investigated MMP-9 and TIMP-1 in induced sputum of asthmatic children and we found that MMP-9 and TIMP-1 are significantly increased in both IA and MA in comparison with $\mathrm{C}$ at both visits 1 and 3 while no differences were present between IA and MA children. We did not find any significant reduction of MMP-9/TIMP-1 molar ratio in these patients in comparison to $\mathrm{C}$, although in adult asthmatic patients the MMP-9/TIMP-1 molar ratio is lower than in control subjects, suggesting a trend toward fibrosis due to the existence of a proteaseantiprotease imbalance (14). The absolute concentration of TIMP-1, and not its molar ratio with MMP-9, has been shown to be associated with persistent wheezing in atopic preschool children (44). Consistently, in our asthmatic children the observed increased concentrations of MMP-9 and TIMP-1 suggests that some proremodeling mediators are present at least in a subgroup of children although in the absence of a protease-antiprotease imbalance. These findings suggest that the remodeling process in some children might be already initiated at the early steps of the disease, although the effects are not yet defined.

IL-8 promotes neutrophil and eosinophil infiltration in the lung (45), leading to chronic inflammation. MMP-9 is released by macrophages, epithelial cells and eosinophils, and TIMP-1 is the major tissue inhibitor secreted in association with MMP-9. Consistently with this, it has already been found a close relationship between levels of IL-8 and MMP-9 in sputum of adult asthmatics as well as a deficiency of antiproteases mechanisms in the same patients (19). In addition, it has been demonstrated that IL- 8 is a substrate for activated MMP-9 and the resultant MMP-9modified IL- 8 molecule is more potent in promoting cell activation (20). Our findings showing a positive correlation between IL-8 and MMP-9 and TIMP-1, and between sputum eosinophils and MMP-9 and TIMP-1, suggest that remodeling mediators may be released simultaneously with inflammatory mediators other than consecutively in asthmatic children. Moreover, the presence of a link between these two events together with the identification of a sub-population of MA with high sputum concentrations of IL-8 which undergoes a higher number of exacerbations during the follow-up suggest that, despite regular treatment, some patients are not controlled in terms of both bronchial inflammation and disease management. The data generated here provide new information for the identification of these specific phenotypes of MA. 
In both adults and children with moderate to severe asthma, it has been demonstrated a persistent activation of $\mathrm{NF}-\kappa \mathrm{B}$ system due to an over-expression of p65 subunits and to an increased p65-DNA complex formation $(6,21)$. We provide evidences that the percentage of sputum cells with a p65 nuclear localization is higher in children with IA and MA than in C at both visits 1 and 3, accordingly with IL-8, MMP-9 and TIMP-1 sputum levels. Moreover, the persistence of the activation of $N F-\kappa B$ pathway in the two groups of asthmatic children, supports the concept that, despite the ongoing ICS therapy, some patients display not completely inhibited molecular mechanisms of airway inflammation. These results confirm and extend some previous observations by our group obtained, at systemic level, in moderate persistent asthmatic children (6). Although we did not identify any correlations between p65 and IL-8, MMP-9 and TIMP-1, the increased p65 nuclear localization in sputum cells suggests that the activation of $\mathrm{NF}-\kappa \mathrm{B}$ system sustains the ongoing production of pro-inflammatory and remodeling mediators also in the airways of these asthmatic children, as previously demonstrated in adults (21).

The use of FeNO measurement and the performance of induced sputum allows to longitudinally assess bronchial inflammation in asthmatic children and to investigate whether the detection of some biomarkers could provide additional information useful in the control and management of the disease. Our findings demonstrating that the number of sputum eosinophils and IL-8 sputum concentrations in IA and MA, correlate with the number of total exacerbations (mild and severe exacerbations) occurred during the follow-up suggest that these inflammatory markers are associated with the disease activity. In addition, in MA, we found that FeNO, eosinophilia, and IL-8 levels significantly correlate with the number of severe exacerbations, indicating the presence of a subclinical inflammation leading to a lack of disease control. According to the levels of these three biomarkers two different subgroups of MA can be identified: the frequent $(>2 / \mathrm{yr})$ and the infrequent ( 0 or $<2 / y r)$ exacerbators. It was already shown, in adults, that a strategy based on the measurement of FeNO or on sputum eosinophils permitted to avoid asthma exacerbations and to reduce inhaled $\mathrm{GC}$ requirement $(11,12)$. The findings that the 'frequent exacerbators' show increased FeNO levels, sputum eosinophilia and increased IL-8 sputum levels extend these previous observations to a pediatric population and further support the link between these markers of inflammation and asthma control and the occurrence of severe exacerbations. Similar results were obtained by Pijnenburg et al. showing that FeNO is an early predicting marker of relapse in asthma after cessation of ICS (46). According to this concept, we observed in MA a positive correlation between IL-8 sputum concentrations and asthma duration. Furthermore, the persistent production of IL-8 may also indicate a greater propensity of the 'frequent exacerbators' to develop severe asthma (16) and to switch to a neutrophilic inflammation which is often associated with this asthma phenotype (47).

In this study, the markers of inflammation (i.e. FeNO, sputum eosinophilia and IL-8) correlate with inflammation-related events, such as asthma exacerbations, while the markers of remodeling do not. These latter markers might likely mirror a future development of the remodeling process.

In the present study, we did not find any differences in terms of $\mathrm{FEV}_{1}$ measurements among the three study groups and the long-term follow-up confirmed the initial clinical assessment of the children. Our finding of lack of significant decrease of $\mathrm{FEV}_{1}$ levels in patients with asthma is consistent with the Bacharier study (48) where this is also valid even in moderate to severe asthmatic children, especially among patients receiving asthma controller therapy. According to this latter study, we found that there is a gradient of decline in $\mathrm{FEV}_{1} / \mathrm{FVC}$ among children with increased asthma severity. These data suggest that $\mathrm{FEV}_{1} / \mathrm{FVC}$ could be more sensitive than $\mathrm{FEV}_{1}$ for the detection of smooth differences of asthma severity. Accordingly, in MA we found an inverse correlation between $\mathrm{FEV}_{1} / \mathrm{FVC}$ and asthma duration, and between $\mathrm{FEV}_{1} / \mathrm{FVC}$ and IL-8 sputum concentrations.

The present study supports the importance to monitor bronchial inflammation to better control asthma in children. We have previously demonstrated that PBMC from some IA children, as well as PBMC from some MA children, were able to release inflammatory cytokines ex vivo. In particular, we showed that moderate asthmatic children can be differentiated into two subgroups according to biologic and clinical parameters, including IL-8 and GM-CSF released by PBMC, and that these markers could be useful to asses the clinical heterogeneity and the control of asthma in children (6). The use of non-invasive procedures allowed us to increase and extend these previous findings by investigating the local bronchial inflammation and by 
evaluating some molecular mechanisms involved in the persistence of inflammation in induced sputum cells.

In conclusion, although the relatively small number of recruited subjects precludes from definitive conclusions on the clinical usefulness of these markers, this study shows that in moderate asthmatic children the inflammatory process is associated with biologic and clinical heterogeneity of the disease, despite regular glucocorticoid treatment. The association between pro-inflammatory and pro-remodeling mediators, could sustain the persistence of inflammation and probably promote the lack of disease control in a subgroup of moderate asthmatics. This study also proposes the need to perform large prospective studies to confirm the importance of measuring specific biomarkers in induced sputum, concomitantly to FeNO analyses, to assess airway inflammation, asthma control, and predictivity of exacerbations in children with asthma.

\section{Acknowledgments}

During the course of this study, Professor A. Maurizio Vignola died from a malignant leukemia. The authors wish to dedicate this work to his memory.

Supported by the Italian National Research Council (CNR), by INSERM-CNR contract, and by EU Framework programme for research, contract no FOOD-CT2004-506378, the GA ${ }^{2}$ LEN project, Global Allergy and Asthma European Network.

\section{References}

1. Vignola AM, Mirabella F, Costanzo G, et al. Airway remodeling in asthma. Chest 2003: 123: 417S-22S.

2. Sont JK, Willems LN, Bel EH, van Krieken JH, VANDEnBRoucke JP, STERK PJ. Clinical control and histopathologic outcome of asthma when using airway hyperresponsiveness as an additional guide to long-term treatment. The AMPUL Study Group. Am J Respir Crit Care Med 1999;159(4 Pt 1):1043-51.

3. VAN DEN TOORN LM, Overbeek SE, DE Jongste JC, Leman K, Hoogsteden HC, Prins JB. Airway inflammation is present during clinical remission of atopic asthma. Am J Respir Crit Care Med 2001: 164: 2107-13.

4. Pohunek P, Warner JO, Turzikova J, Kudrmann J, Roche WR. Markers of eosinophilic inflammation and tissue re-modelling in children before clinically diagnosed bronchial asthma. Pediatr Allergy Immunol 2005: 16: 43-51.

5. Marguet C, Dean tP, Basuyau JP, Warner JO. Eosinophil cationic protein and interleukin-8 levels in bronchial lavage fluid from children with asthma and infantile wheeze. Pediatr Allergy Immunol 2001: 12: 2733.

6. La Grutta S, Gagliardo R, Mirabella F, et al. Clinical and biological heterogeneity in children with moderate asthma. Am J Respir Crit Care Med 2003: 167: $1490-5$
7. Zacharasiewicz A, Wilson N, Lex C, et al. Clinical use of noninvasive measurements of airway inflammation in steroid reduction in children. Am J Respir Crit Care Med 2005: 171: 1077-82.

8. Berlyne GS, Parameswaran K, Kamada D, EfthIMIADIS A, Hargreave FE. A comparison of exhaled nitric oxide and induced sputum as markers of airway inflammation. J Allergy Clin Immunol 2000: 106: 63844.

9. Wilson NM, James A, Uasuf C, et al. Asthma severity and inflammation markers in children. Pediatr Allergy Immunol 2001: 12: 125-32.

10. Sorva R, Metso T, Turpeinen M, Juntunen-BackMAN K, BJÖRKSTEN F, HAAHTELA T. Eosinophil cationic protein in induced sputum as a marker of inflammation in asthmatic children. Pediatr Allergy Immunol 1997: 8: $45-50$.

11. Green RH, Brightling CE, McKenna S, et al. Asthma exacerbations and sputum eosinophil counts: a randomised controlled trial. Lancet 2002: 360: 1715-21.

12. Pijnenburg MW, Bakker EM, Hop WC, De Jongste JC. Titrating steroids on exhaled nitric oxide in children with asthma: a randomized controlled trial. Am J Respir Crit Care Med 2005: 172: 831-6.

13. Profita M, Gagliardo R, Di Giorgi R, et al. Biochemical interaction between effects of beclomethasone dipropionate and salbutamol or formoterol in sputum cells from mild to moderate asthmatics. Allergy 2005: 60: 323-9.

14. Vignola AM, Riccobono L, Mirabella A, et al. Sputum metalloproteinase-9/tissue inhibitor of metalloproteinase- 1 ratio correlates with airflow obstruction in asthma and chronic bronchitis. Am J Respir Crit Care Med 1998: 158: 1945-50.

15. Shannon J, Ernst P, Yamauchi Y, et al. Differences in airway cytokine profile in severe asthma compared to moderate asthma. Chest 2008: 133: 420-6.

16. Gagliardo R, Chanez P, Vignola AM, et al. Glucocorticoid receptor alpha and beta in glucocorticoid dependent asthma. Am J Respir Crit Care Med 2000: 162: 7-13.

17. Wilson JW, Li X, PAIN MC. The lack of distensibility of asthmatic airways. Am Rev Respir Dis. 1993: 148: $806-9$.

18. Ohno I, Ohtani H, Nitta Y, et al. Eosinophils as a source of matrix metalloproteinase-9 in asthmatic airway inflammation. Am J Respir Cell Mol Biol 1997: 16: 212-9.

19. Simpson JL, Scott RJ, Boyle MJ, Gibson PG. Differential proteolytic enzyme activity in eosinophilic and neutrophilic asthma. Am J Respir Crit Care Med 2005: 172: 559-65.

20. Van den Steen Pe, Proost P, Wuyts A, Van Damme J, OpdenAKKer G. Neutrophil gelatinase B potentiates interleukin-8 tenfold by aminoterminal processing, whereas it degrades CTAP-III, PF-4, and GRO-alpha and leaves RANTES and MCP-2 intact. Blood 2000: 96: 2673-81.

21. Gagliardo R, Chanez P, Mathieu M, et al. Persistent activation of nuclear factor-kappaB signaling pathway in severe uncontrolled asthma. Am J Respir Crit Care Med 2003: 168: 1190-8.

22. Rothwarf DM, Karin M. The NF-kappa B activation pathway: a paradigm in information transfer from membrane to nucleus. Sci STKE 1999: 1999:RE1.

23. Gum R, Lengyel E, Juarez J, et al. Stimulation of 92$\mathrm{kDa}$ gelatinase $\mathrm{B}$ promoter activity by ras is mitogen- 


\section{Gagliardo et al.}

activated protein kinase kinase 1-independent and requires multiple transcription factor binding sites including closely spaced PEA3/ets and AP-1 sequences. J Biol Chem 1996: 271: 10672-80.

24. Wilczynska KM, Gopalan SM, Bugno M, et al. A novel mechanism of tissue inhibitor of metalloproteinases-1 activation by interleukin-1 in primary human astrocytes. J Biol Chem 2006: 281: 34955-64.

25. Global Initiative for Asthma (GINA). Workshop report, Global Strategy for Asthma Management and Prevention, 2005: National Institutes of Health, National Heart, Lung and Blood Institute. NHI Publication N. 02-3659.

26. Miller MR, Hankinson J, Brusasco V, et al. Standardisation of spirometry. Eur Respir J 2005: 26: 319-38.

27. Dreborg S. Skin testing. The safety of skin tests and the information obtained from using different methods and concentrations of allergen.. Allergy 1993: 48: 473-5.

28. Eid N, Morton R, Olds B, Clark P, Sheikh S, LOONEY S. Decreased morning serum cortisol levels in ticasone propionate. Pediatrics 2002: 109: 217-21.

29. Hagg E, Asplund K, Lithner F. Value of basal plasma cortisol assays in the assessment of pituitary-adrenal insufficiency. Clin Endocrinol 1987: 26: 221-6.

30. Pauwels RA, Lofdahl CG, Postma DS, et al. Effect of inhaled formoterol and budesonide on exacerbations of asthma. Formoterol and Corticosteroids Establishing Therapy (FACET) International Study Group. N Engl J Med 1997: 337:1405-11.

31. in't Veen JC, Smits HH, Hiemstra PS, Zwinderman AE, STERK PJ, Bel EH. Lung function and sputum characteristics of patients with severe asthma during an induced exacerbation by double-blind steroid withdrawal. Am J Respir Crit Care Med 1999: 160: 93-9.

32. ATS/ERS. Recommendations for standardized procedures for the online and offline measurement of exhaled lower respiratory nitric oxide and nasal nitric oxide. American Thoracic Society, European Respiratory Society: 171: 912-30.

33. Jones PD, Hankin R, Simpson J, Gibson PG, Henry RL. The tolerability, safety, and success of sputum induction and combined hypertonic saline challenge in children. Am J Respir Crit Care Med 2001: 164: 1146-9.

34. Hargreave FE, Popov T, Kidney J, Dolovich J. Sputum measurements to assess airway inflammation in asthma. Allergy. 1993: 48: 81-3.

35. Baraldi E, Azzolin NM, Zanconato S, Dario C, ZaCCHEllo F. Corticosteroids decrease exhaled nitric oxide in children with acute asthma. J Pediatr 1997: 131: 381-5.

36. Hamid Q, Springall DR, Riveros-Moreno V, et al. Induction of nitric oxide synthase in asthma. Lancet 1993: 342: 1510-3.
37. Kharitonov SA, Gonio F, Kelly C, Meah S, Barnes PJ. Reproducibility of exhaled nitric oxide measurements in healthy and asthmatic adults and children. Eur Respir J 2003: 3: 433-8.

38. Fritsch M, UxA S, HoraK F JR, et al. Exhaled nitric oxide in the management of childhood asthma: a prospective 6-months study. Pediatr Pulmonol 2006: 41: 855-62.

39. Buchvald F, Eiberg H, Bisgaard H. Heterogeneity of FeNO response to inhaled steroid in asthmatic children. Clin Exp Allergy 2003: 33: 1735-40.

40. Robroeks CM, van DE Kant KD, Jöbsis Q, et al. Exhaled nitric oxide and biomarkers in exhaled breath condensate indicate the presence, severity and control of childhood asthma. Clin Exp Allergy 2007: 37: 1303-11.

41. Fukakusa M, Bergeron C, Tulic MK, et al. Oral corticosteroids decrease eosinophil and CC chemokine expression but increase neutrophil, IL-8, and IFNgamma-inducible protein 10 expression in asthmatic airway mucosa. J Allergy Clin Immunol 2005: 115: 280-6.

42. Norzila MZ, Fakes K, Henry RL, Simpson J, Gibson PG. Interleukin-8 secretion and neutrophil recruitment accompanies induced sputum eosinophil activation in children with acute asthma. Am J Respir Crit Care Med 2000: 161: 769-74.

43. Cokuğraş H, Akçakaya N, Seçkin S, CamcioĞlu Y, SARIMURAT N, AKSOY F. Ultrastructural examination of bronchial biopsy specimens from children with moderate asthma. Thorax 2001: 56: 25-9.

44. Erlewyn-Lajeunesse MD, Hunt LP, Pohunek P, et al. Bronchoalveolar Lavage MMP-9 and TIMP-1 in Preschool Wheezers and Their Relationship to Persistent Wheeze. Pediatr Res 2008: 64: 194-9.

45. Lampinen M, Carlson M, Håkansson LD, Venge P. Cytokine-regulated accumulation of eosinophils in inflammatory disease. Allergy 2004: 59: 793-805.

46. Pijnenburg MW, Hofhuis W, Hop WC, De Jongste JC. Exhaled nitric oxide predicts asthma relapse in children with clinical asthma remission. Thorax 2005: 60: 215-8.

47. Wenzel SE, Schwartz LB, Langmack EL, et al. Evidence that severe asthma can be divided pathologically into two inflammatory subtypes with distinct physiologic and clinical characteristics. Am J Respir Crit Care Med 1999: 160: 1001-8.

48. Bacharier LB, Strunk RC, Mauger D, White D, Lemanske RF $J_{R}$, Sorkness CA. Classifying asthma severity in children: mismatch between symptoms, medication use, and lung function. Am J Respir Crit Care Med 2004: 170: 426-32. 